\title{
La suburbanización del Sunbelt estadounidense tras la crisis del petróleo. El crecimiento como ideología y el debate medioambiental
}

Carlos García-Vázquez. Universidad de Sevilla, Sevilla, España.

RESUMEN | El artículo analiza el desarrollo del modelo suburbano en las ciudades del Sunbelt en las décadas posteriores a la crisis del petróleo de 1973, especialmente 1980 y 1990. Paradójicamente, en un momento en que la citada crisis y la publicación del Informe Meadows expandían la sensibilidad ecologista por el mundo, el Sunbelt seguía apostando por el modelo de desarrollo suburbano, ya desacreditado como insostenible. En el artículo se estudian las razones que avalaron su éxito, principalmente la incorporación de áreas de oficinas a los nuevos suburbios, así como la crisis medioambiental que desató a finales de 1990. Igualmente se analizan los intentos del movimiento "smart growth" por corregir sus aspectos más insostenibles y los contraargumentos de los defensores del modelo suburbano. El artículo defiende que el consenso de la sociedad del Sunbelt en favor de este cuenta con una base ideológicoeconómica.

PALABRAS CLAVE | calidad ambiental, crecimiento urbano, transformaciones socioterritoriales.

ABSTRACT | This article analyzes the development of the suburban model in the Sunbelt cities in the decades after the 1973 oil crisis, especially in the 1980s and 1990s. Paradoxically, in a moment when the economic crisis and the publication of the Meadows Report were expanding the ecologist sensibility all over the world, the Sunbelt continued supporting the suburban model, already discredited as unsustainable. The article studies the reasons of this success, mainly the insertion of office uses in the new suburbs, as well as the resulting environmental crisis at the end of the 1990s. It also analyzes the proposals of the "smart growth" movement in order to correct the most unsustainable aspects of the suburban model, and the counter-arguments of its defenders. Finally, the article considers that the wide social consensus that this model has in the Sunbelt has an ideological and economic basis.

KEYWORDS | environmental quality, urban growth, socio-territorial transformations. 


\section{Introducción}

Con el término "Sunbelt" o "Cinturón del Sol" se designa la franja de Estados Unidos comprendida entre el paralelo 37 y la frontera con México. En ella se ubican catorce estados: la mitad sur de California, Arizona, Nuevo México, Texas, Oklahoma, Arkansas, Luisiana, Tennessee, Mississippi, Alabama, Georgia, Carolina del Norte, Carolina del Sur y Florida. También decenas de ciudades que, tras la Segunda Guerra Mundial y siguiendo un modelo previamente definido por Los Ángeles, apostaron por el crecimiento suburbano: Phoenix, Albuquerque, Dallas, Houston, Atlanta, Miami, etcétera.

Desde la Segunda Guerra Mundial, en Estados Unidos se ha producido una explosión urbanizadora que multiplica escalarmente el desarrollo demográfico. De 1950 a la actualidad, el número de habitantes se ha triplicado, mientras que la superficie construida se ha quintuplicado; y en la década de 1990 el consumo de territorio era un 50\% superior al crecimiento demográfico. Por lo que respecta al Sunbelt, entre 1970 y 1990 la población de Los Ángeles aumentó un 45\%, mientras que su superficie lo hizo un $300 \%$ (figura 1); y en Florida, el suelo urbanizado se incrementó un 80\% entre 1974 y 1984, el doble que la población. Lo que explica este desfase es el modelo urbanístico que las metrópolis norteamericanas, y muy especialmente las del Sunbelt, han elegido para materializar su crecimiento: el suburbano. Frente a la compacta ciudad tradicional, este modelo, basado en la baja densidad, exige mucho más consumo de territorio. Los Ángeles, por ejemplo, necesita siete veces más suelo que Brooklyn para albergar el mismo número de habitantes, y Phoenix el doble que Detroit. Jane H. Kay (1997) comentaba: "Habiendo pasado de 44 a $1.100 \mathrm{~km}^{2}$ de superficie en cuarenta años, cuenta actualmente con diez veces el número de hectáreas de la malla de Manhattan. Con 48 kilómetros en su dimensión más amplia, Phoenix tiene aproximadamente la longitud de la ciudad más poblada de la nación y más de dos tercios de su anchura" (p. 58; traducción y adaptación del autor).

Numerosa literatura sobre el tema ha demostrado que la apuesta de Estados Unidos por el modelo suburbano tiene profundas raíces histórico-culturales (Fishman, 1987; Hayden, 2003). Tras la Segunda Guerra Mundial, 141 millones de estadounidenses pudieron acceder a una vivienda digna gracias a dicho modelo. El bajo precio del suelo, unido a las avanzadas técnicas de prefabricación, hicieron posible que dos tercios de la población adquiriera una casa (un 60\% más que en la mayoría de los países europeos). Se trataba, además, de viviendas con estándares de calidad y superficie bastante superiores a los del Viejo Continente; y de urbanizaciones con excelentes escuelas, centros comerciales, de ocio, etcétera. Los suburbios de posguerra fueron clave para alcanzar el nivel de vida del que actualmente disfruta el estadounidense medio. Ello explica que muchos de ellos identifiquen la residencia unifamiliar con el American way of life. De acuerdo con una encuesta llevada a cabo en 1999 , el $83 \%$ opinaba que era el mejor modelo urbano posible. 
FIGURA I Comparación de los crecimientos demográfico y territorial en cinco ciudades de Estados Unidos entre 1970 y 1990. De ellas, tan solo Los Ángeles pertenece al Sunbelt

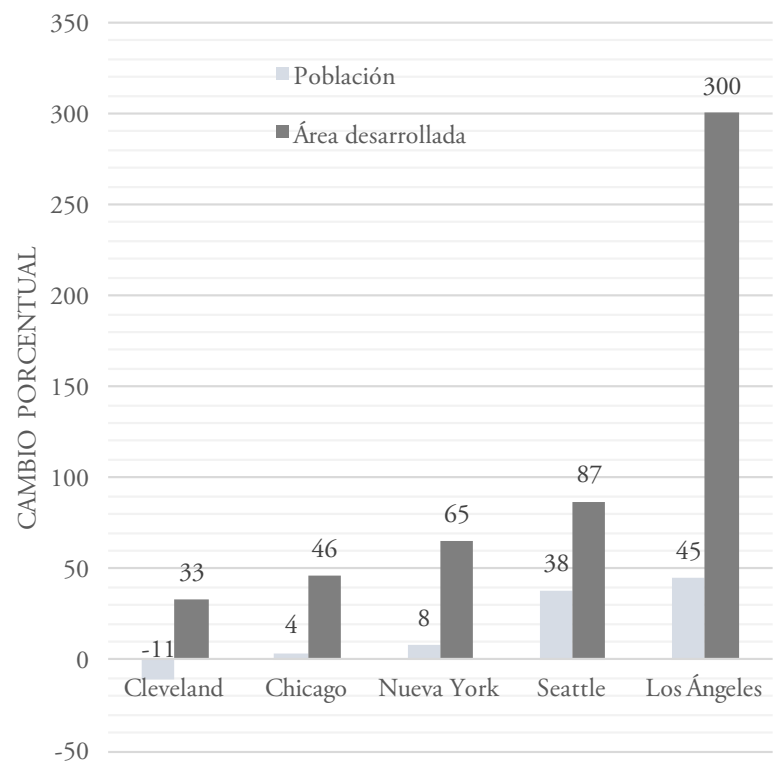

FUENTE H. DIAMOND \& P. NOONAN (I 996). LAND USE IN AMERICA. WASHINGTON DC: ISLAND PRESS (TRADUCCIÓN Y ADAPTACIÓN DEL AUTOR)

El presente artículo defiende que, en el caso del Sunbelt, además de las citadas razones histórico-culturales, el modelo suburbano cuenta con una fundamentación ideológica. En esta zona crecer es una ideología, una forma de pensar la economía, la sociedad, el territorio y la ciudad. El lema es growth is good (Abbott, 1993, p. 181). Grady Gammage Jr. (1999) comentaba sobre Phoenix: “Desde sus inicios, la fijación de esta comunidad ha sido sobrevivir y crecer en el desierto. Crecer para conseguir agua. Crecer para competir con California. Crecer para vender tierra. Crecer para demostrar que este es un lugar espléndido. Una vez que la máquina del crecimiento se puso en marcha, no quisimos parar (...). La única imagen de sí misma que Phoenix ha desarrollado es la del crecimiento" (p. 59; traducción y adaptación del autor) (figura 2).

La “ideología del crecimiento" tiene hondas raíces históricas. Nació en Los Ángeles a comienzos del siglo xx. Entre 1900 y 1930 esta ciudad explosionó demográfica y territorialmente. Los 170.000 habitantes de su condado se multiplicaron por trece, hasta alcanzar los 2.209.000. En esas décadas el verdadero motor económico fue el desarrollo urbano, un hecho novedoso desde el punto de vista histórico. Si hasta entonces el incremento demográfico y territorial de las ciudades había sido consecuencia de su crecimiento económico, se inauguraba ahora una época donde el desarrollo urbano atraía a la vez empleos y residentes; es decir, donde expandirse territorialmente era un motor económico en sí mismo. Esta fórmula, 
posteriormente denominada "economía del ladrillo", se propagó por el Sunbelt en la década de 1930, cuando se teorizó sobre el éxito de Los Ángeles. La sancionaron las políticas estatales (que concentraron sus inversiones en la construcción de infraestructuras urbanas) y la alentó la prensa (que alardeaba de "superciudades que explotaban bajo los cielos sureños”) (Bernard \& Rice, 1983). Tras ambas estaban las elites económicas. Tal como afirmaba H. V. Savitch: "Banqueros, agentes inmobiliarios, abogados, promotores, periódicos, sindicatos, cámaras de comercio e innumerables otros actores conducen la máquina del crecimiento. Banqueros y agentes inmobiliarios obtienen beneficios apoyando a los promotores, mientras que periódicos, comercios y sindicatos ansían construir y vender cuanto más mejor" (en Squires, 2002, pp. 146; traducción y adaptación del autor).

FIgURA 2 | Crecimiento territorial de Phoenix entre 1881 y 1998

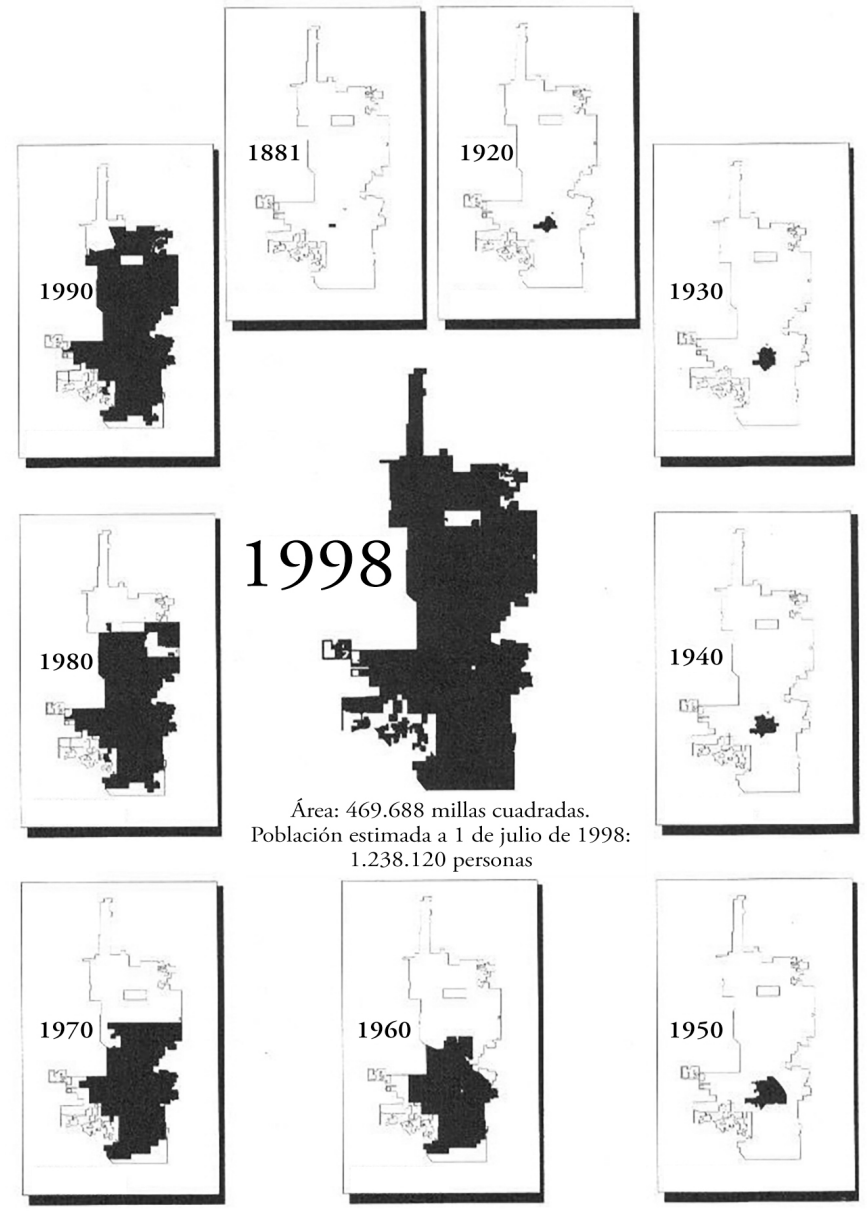

FUente City of Phoenix planning Department. EN G. GAMMAge (JR.) (I999). Phoenix in PERSPECTIVE. REFLECTIONS ON DEVELOPING THE DESERT. TEMPE, AZ: ARIZONA STATE UNIVERSITY, P. 37 (TRADUCCIÓN Y ADAPTACIÓN DEL AUTOR) 
El papel subsidiario del Estado y los medios de comunicación con respecto a los poderes económicos locales era una tradición en las ciudades del Sunbelt. Desde su fundación, cuando no eran más que pequeñas comunidades habitadas por colonos agrícolas, se vieron obligadas a rivalizar entre sí por acaparar recursos y mercados. El liderazgo de esta concurrencia fue asumido por los promotores privados. Fueron ellos, y no los poderes públicos, los que las dotaron de las infraestructuras necesarias para acceder a los recursos naturales y a los mercados de consumidores. ${ }^{1}$ En la década de 1950, esta tradición de liderazgo empresarial devino en un consenso social que puso numerosos ayuntamientos en manos de los hombres de negocio y elites profesionales locales. El desarrollo urbano de Dallas pasó a ser controlado por la Citizen's Charter Association, un grupo de empresarios; en San Antonio, el 94\% de los concejales elegidos desde 1955 pertenecen a la asociación empresarial Good Government League (Perry \& Watkins, 1977, p. 185); algo similar ocurrió en Phoenix con otro colectivo de promotores, el Phoenix 40.

Esta ciudad ofrece un buen ejemplo de las razones que sustentan el apoyo ciudadano a la ideología del crecimiento:

Al fin y al cabo, Phoenix ha demostrado ser una ciudad con una sola industria, al igual que Houston o Denver (...). La industria no es el petróleo, por supuesto. Es crecer. Si uno suma los obreros de la construcción, los empleados de las inmobiliarias, los vendedores de seguros, arquitectos, tasadores, banqueros y economistas, así como funcionarios públicos relacionados con el mundo de la construcción, totalizan el $20 \%$ de la fuerza laboral. Es decir, es el crecimiento, más que cualquier otro elemento, el que genera la ilusión de prosperidad en el condado de Maricopa (J. Laing, en Gammage, 1999, p. 58; traducción y adaptación del autor).

Gracias a la economía del ladrillo, y a la importancia del petróleo como sector productivo, el Sunbelt salió indemne de la crisis del petróleo que asoló Occidente a partir de 1973. A pesar de la depresión económica que se abatía sobre el resto del país, entre 1980 y 1985 se crearon en Phoenix 174.000 empleos, lo que convirtió a Arizona en el primer estado en generación de puestos de trabajo. Phoenix podía permitirse ironizar sobre la crisis. ${ }^{2}$ En aquel momento era la ciudad más próspera de Estados Unidos: había ganado 330.000 habitantes en cinco ańos (Luckingham, 1989, pp. 221-268).

Desde finales de la década de 1970, numerosos autores han vuelto su mirada hacia el Sunbelt, convencidos de que allí se estaba fraguando un modelo de ciudad sin referentes históricos. Pioneros fueron Reyner Banham (1971), David C. Perry y Alfred

1 Houston es un buen ejemplo de ello. En sus inicios, esta ciudad, que distaba unos 80 kilómetros del Golfo de México, vivía a la sombra de su vecina Galveston que, por su situación en la costa y su puerto natural, se perfilaba como futuro centro regional. En 1909 los empresarios de Houston pusieron en marcha una iniciativa para construir un puerto interior en el pantano de Buffalo que conectara la ciudad con la costa. Emitieron bonos para financiarlo y constituyeron lobbies de presión en Washington para conseguir fondos adicionales del gobierno federal. En 1914 se inauguraba el Houston Ship Channel, al que se trasladaron las compañías petroleras de Texas, cuyas refinerías estaban situadas en otras localidades (en Perry \& Watkins, 1977, pp. 109-128).

2 Bien distinto ha sido el caso de la crisis de 2008, que ha afectado profundamente a las ciudades del Sunbelt debido a que uno de sus epicentros estaba en el sector inmobiliario. 
J. Watkins (1977), Ada Louise Huxtable (1978) y Richard M. Bernard (1983). Entre los estudios posteriores destacan los de Robert B. Fairbanks y Katheleen Underwood (1990), Matthew D. Lassiter (2007) y Carlos García Vázquez (2011).

Los objetivos de este artículo son:

- Determinar cuáles fueron los motores del modelo suburbano en el Sunbelt en las décadas de 1980 y 1990, cuando el ecologismo ya se había extendido y consolidado en el resto del planeta.

- Analizar las propuestas que se plantearon para mitigar la crisis medioambiental que se derivó de dicho modelo a finales de 1990, su implementación y su posterior declive.

- Comprender las razones de la persistencia de la ideología del crecimiento en el Sunbelt.

La metodología utilizada se basará en el análisis histórico de la evolución del tema en las últimas décadas, comenzando en los años 1980, cuando el ecologismo se expandió a nivel global, y terminando en los inicios del presente siglo, cuando se da por consolidado el modelo económico tardocapitalista. Para ello se estudiarán: las bases conceptuales que sustentan la coalición entre el modelo suburbano y la ideología del crecimiento en el Sunbelt; las propuestas que pretendieron corregir dicho modelo en las décadas mencionadas ("smart growth" y "edge city"); las razones que explican el declive de ambas, incidiendo en los argumentos esgrimidos por los defensores de la ideología del crecimiento; y el desemboque en la situación actual, la denominada "edgeless city".

\section{La redefinición del modelo suburbano en las décadas de 1980 y 1990}

En las dos últimas décadas del siglo xx, la población de las áreas suburbanas estadounidenses aumentó diez veces más rápidamente que la de los centros históricos. Según la Oficina del Censo de Estados Unidos, ${ }^{3}$ entre 1988 y 1996 estos perdieron 2,9 millones de habitantes, mientras que los suburbios aumentaron a un ritmo medio de tres millones anuales. En 1996, mientras 800.000 personas optaban por mudarse a los cascos históricos, 2,7 millones decidían abandonarlos en dirección a los suburbios (Squires, 2002, p. 6). En el Sunbelt, su crecimiento triplicó el de los centros urbanos. Si en 1965 tan solo el 36,8\% de los habitantes de la aglomeración Dallas-Fort Worth vivía en suburbios, en 2003 lo hacía el 69,0\%. En ese mismo periodo de tiempo, la población suburbana de Houston pasó del 26,2 al 61,2\% y la de Las Vegas del 25,5 al 63,6\%.

Lo anterior explica que las ciudades del Sunbelt se encuentren actualmente entre las más suburbanas del planeta. ${ }^{4}$ Los censos demuestran que el punto de inflexión que acentuó la línea ascendente de lo que en las décadas previas se había denominado "suburbia" se produjo en los años 1970, coincidiendo con la crisis del

4 Según el World Urban Areas de 2017, informe anual del think tank Demographia, tan solo Brisbane (Australia) y Quebec (Canadá) pueden competir con ellas. 
petróleo (1973) y la publicación del Informe Meadows (1973), y se fortaleció en la década siguiente, en plena efervescencia del Informe Bruntland (1987). Paradójicamente, mientras que estos dos documentos expandían por el mundo la sensibilidad medioambiental, las ciudades del Sunbelt reforzaban su apuesta por un modelo de crecimiento urbano demostradamente insostenible.

Lo que explica esta paradoja es el hecho de que en esas dos décadas tomó forma y se consolidó el tardocapitalismo. Numerosos autores (Benfield, Raimi \& Chen, 1999, p. 14; Castells, 1995; Fishman, 1987, p. 195; Lang, 2003, p. 55) apuntan a un fenómeno claramente vinculado con la globalización de la economía: al tradicional atractivo residencial del suburbio se sumó su potencial como enclave laboral; más concretamente, como sede de edificios de oficinas (figura 3). Muchas razones habían impulsado a las empresas a abandonar los centros históricos: altísimos precios de suelo, deterioro ambiental y social, inseguridad, etcétera. Las tecnologías de la información lo hicieron posible, al permitirles seguir en contacto con clientes y vendedores a pesar de la distancia física.

FIgURA 3 | Direcciones del crecimiento en el área metropolitana de Atlanta entre 1980 y 1998. Personas y empleos

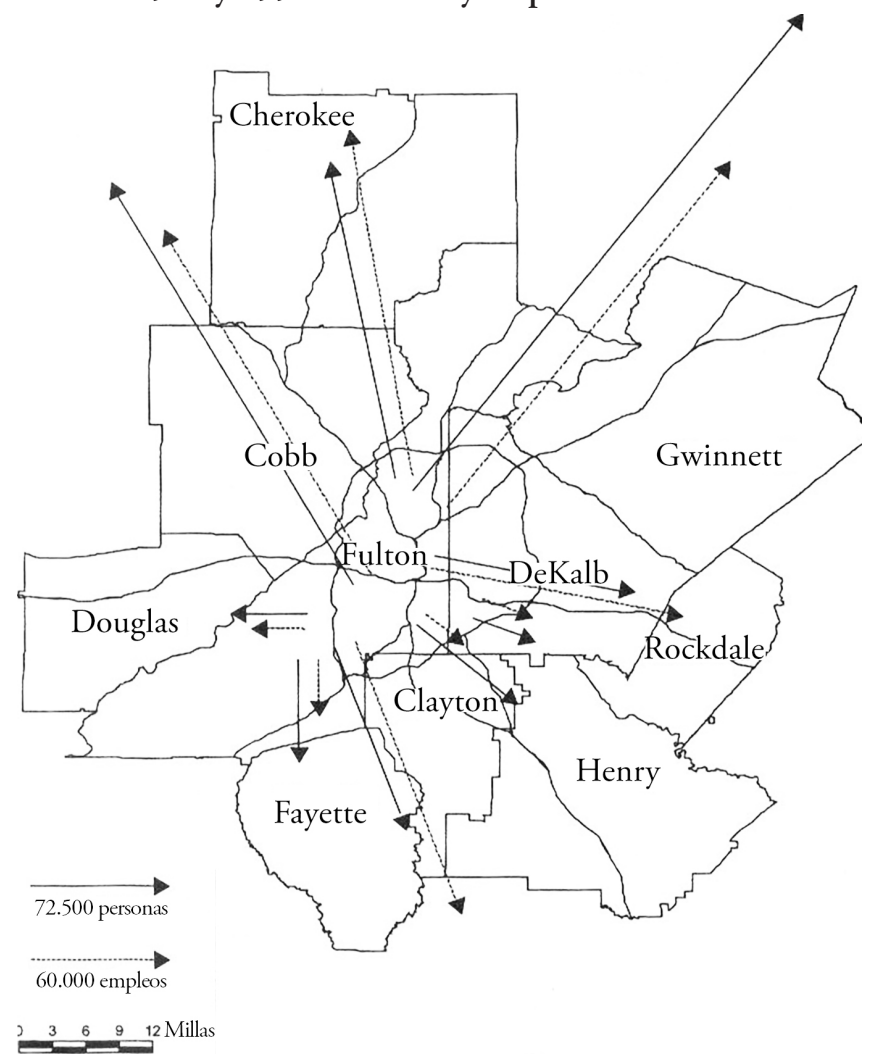

FUENTE B. CALVERT. EN L. KEATING (2OOI). ATLANTA. RACE, CLASS AND URBAN EXPANSION. FILADELFIA, PA: TEMPLE UNIVERSITY PRESS, P. IO (TRADUCCIÓN Y ADAPTACIÓN DEL AUTOR). 
Las multinacionales trasladaron al suburbio lo que se conoce como "back office", trabajos rutinarios que no requerían de personal altamente cualificado ni de entornos arquitectónicos y urbanos representativos (como, por ejemplo, los servicios telefónicos de atención al cliente). También optaron por el suburbio compañías pequeñas y medianas que no podían hacer frente a los elevados costos de localización en los centros urbanos, así como empresas de servicios sociales y de servicios al consumidor que necesitaban estar cerca de las residencias de sus clientes. A todas ellas les siguieron gabinetes de abogados, ingenieros, brokers, bancos, etcétera (Castells, 1995). Ello explica que el 95\% de los 15 millones de empleos que se crearon en el sector de oficinas entre 1980 y 1990 estuviera en los suburbios. ${ }^{5}$ Una vez más, esta realidad era especialmente intensa en el Sunbelt: si en 1999 el espacio de oficinas suburbial de las trece áreas metropolitanas más importantes de Estados Unidos representaba el 56,3\% del total, en Houston suponía el 77\%, en Phoenix el 80\% y en Miami el 82,4\% (Lang, 2003, p. 55). Para Robert Fishman (1987), este fenómeno significaba el final del proceso de suburbanización y la entrada en otra fase, el advenimiento de un nuevo tipo de ciudad donde la periferia se independizaba del centro. Ante esta realidad, teóricos como Rob Kling, Spencer Olin y Mark Oster (1995) defendían que, más que como "sub-urbia", habría que designarla "postsuburbia", un entorno donde lo que antes era la sustancia (el centro urbano) se había convertido en mero accidente y donde la excepción (el suburbio) se había transformado en regla.

El vigor de postsuburbia se puso de manifiesto en su explosiva demografía. En los años 1990 apareció un neologismo que intentaba ponerle nombre: "boomburb". Con él se calificaban a los suburbios de más de 100.000 habitantes cuyas tasas de crecimiento superaban los dos dígitos. Se estimaba que en Estados Unidos había más de cincuenta (Robert E. Lang y Patrick A. Simmons en Katz \& Lang, 2003, pp. 101-115). Destacaban los que rodeaban Phoenix. En los últimos cincuenta años, la población de Scottsdale había aumentado un 1.922\% (figura 4), la de Tempe un $1.964 \%$, la de Glendale un 2.575\% y la de Chandler un 4.548\%. Mesa había necesitado más de un siglo para atraer a sus primeros cien mil residentes (de 1878 a 1997) y menos de una década para sumar los siguientes cien mil. A principios del siglo xxI, el $43 \%$ de los habitantes del área metropolitana de Phoenix vivía en estos boomburbs, que competían con la ciudad madre por atraer empresas y por seguir creciendo.

Lógicamente, también en las áreas suburbanas el crecimiento territorial multiplicaba el demográfico. A ello contribuían varios factores. En primer lugar, políticas administrativas fundamentadas en la ideología del crecimiento. Las legislaciones de los estados del Sunbelt contemplan un instrumento que la facilitaba: el derecho de anexión (Abbott, 1981). Gracias a él, una ciudad puede agregarse territorios colindantes a su término municipal. Tras la anexión, la ciudad central y el suburbio adicionado comparten un mismo ayuntamiento y una misma jurisdicción; y la primera se compromete a dotar al segundo de servicios e infraestructuras públicas. En segundo lugar, las topografías planas propias de la zona, llanuras y desiertos 
que no oponían límites naturales al crecimiento. Es lo que ocurría en Houston: situada en una inmensa pradera, podía extenderse en cualquier dirección, con la única demarcación del Golfo de México. Algo similar era el caso de Atlanta, solo que allí la línea de costa quedaba a cientos de kilómetros de distancia. Y aún más extremo era el de Phoenix. Su entorno natural, el desierto de Sonora, le permitía una expansión potencialmente casi infinita.

\section{Figura 4 | Sottsdale, un “boomburb” de Phoenix}

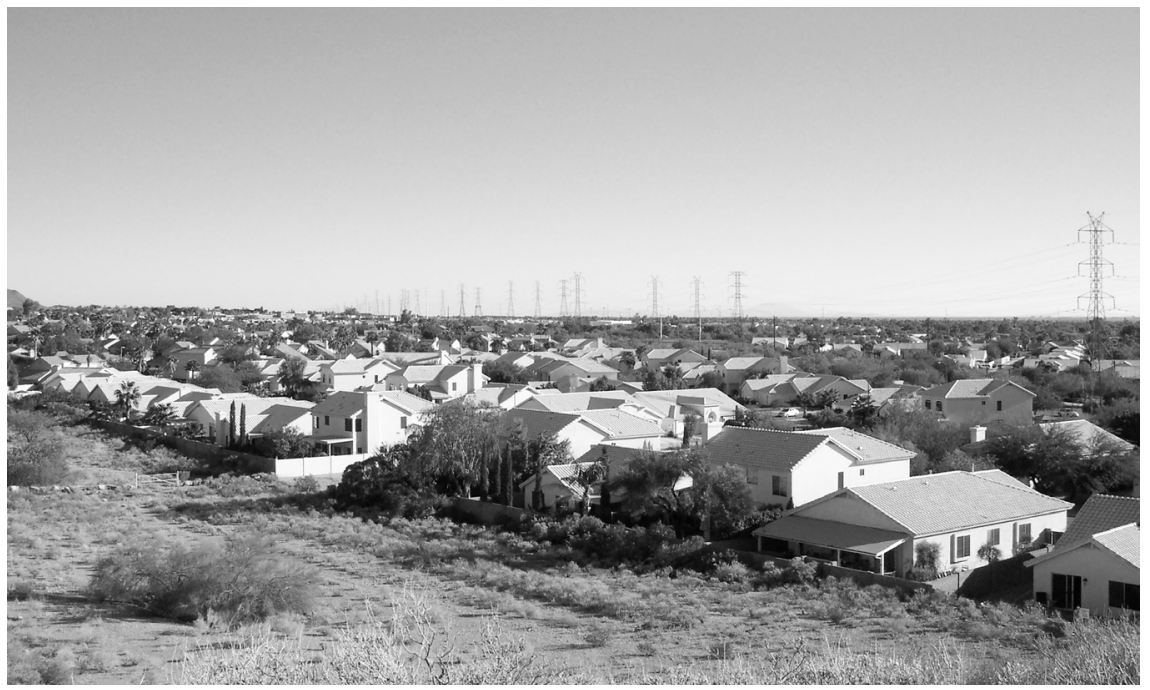

FUENTE ELABORACIÓN PROPIA

La confluencia de estos factores topográficos y político-administrativos favoreció un gigantismo territorial sin precedentes. Según los datos de la Oficina del Censo, en el año 2000 las cinco ciudades más grandes de Estados Unidos en términos de superficie estaban en el Sunbelt: Houston con $1.398 \mathrm{~km}^{2}$, Los Ángeles con $1.215 \mathrm{~km}^{2}$, Phoenix con $1.087 \mathrm{~km}^{2}$, Dallas-Fort Worth con $886 \mathrm{~km}^{2}$ y San Diego con $838 \mathrm{~km}^{2}$ (la sexta era Nueva York, con $800 \mathrm{~km}^{2}$ ) (Katz \& Lang, 2003). Todas ellas habían crecido exponencialmente en las décadas anteriores. Paradigmático era el caso de Phoenix (figura 2). Los $25 \mathrm{~km}^{2}$ de 1940 se convirtieron en 485 en 1960 y en 1.100 en 1997. Dentro de sus límites cabían París, San Francisco, Roma, Manhattan y Washington (Gammage, 1999, pp. 39, 49, 63). Pero la estrella del crecimiento territorial era Atlanta. En 1999, la revista Time la declaró el asentamiento humano que más rápidamente crecía de la historia (1,2 hectáreas por hora) (Lacayo, 1999). Su dimensión norte-sur, que era de 104 kilómetros en 1990, saltó a los 177 en 1998. Atlanta ostentaba, además, otro récord: era una de las ciudades más suburbanas de Estados Unidos (en 2000, el 88,1\% de sus habitantes vivía en suburbios). 


\section{La factura de la ideología del crecimiento: la crisis medioambiental}

El indudable bienestar social que la ideología del crecimiento garantizó a las ciudades del Sunbelt durante las décadas de 1980 y 1990 se estaba cobrando dos importantes facturas: la económica y la medioambiental. La primera es fácilmente cuantificable. El modelo suburbano es mucho más caro que el de la ciudad compacta: a más espacio entre casas y edificios, más kilómetros de carreteras, de redes de agua, de alcantarillado, etcétera. El importe de las infraestructuras necesarias para abastecer una vivienda unifamiliar aislada duplica el de un apartamento de un bloque plurifamiliar urbano. Si tenemos en cuenta que, en las décadas que nos ocupan, cada kilómetro de alcantarillado costaba unos 125.000 dólares, que cada kilómetro de autopista costaba 2,5 millones de dólares y que las infraestructuras hay que mantenerlas, nos haremos una idea aproximada de la envergadura de esta primera factura. A pesar de ello, el modelo suburbano es rentable para los promotores, ya que el suelo es barato y gran parte de esas infraestructuras (autopistas, depuradoras, colegios, etcétera) son financiadas por la administración pública. Se calcula que por cada dólar de impuestos que un suburbanita paga a las arcas del estado, demanda de estas entre 1,12 y 1,36 dólares (Benfield, Raimi \& Chen, 1999, pp. 89-116; Gillham, 2002, pp. 123-132).

La segunda factura, la medioambiental, es más difícil de cuantificar. A la dependencia del automóvil, la polución ambiental, el gasto energético, el calentamiento de la atmósfera, la destrucción de la capa de ozono, etcétera, se suman otros costes. Uno de ellos es la dilapidación del territorio. Estados Unidos urbaniza 150 hectáreas cada hora. Si las actuales tasas de crecimiento continúan, la superficie edificada se duplicará en aproximadamente cincuenta ańos. El modelo suburbano es en gran medida responsable de esta sangría, ya que necesita cinco veces más territorio que la ciudad tradicional para albergar un millón de habitantes $\left(1.060 \mathrm{~km}^{2}\right.$ frente a $220 \mathrm{~km}^{2}$ ). Parte de ese territorio son bosques y zonas húmedas, donde vive el $50 \%$ de las especies animales del país. Pero la mayoría es superficie agrícola. Estados Unidos pierde cada ańo un millón de hectáreas de tierras cultivables. De ellas, 240.000 se encuentran entre las más productivas de la nación (donde se recolecta el 79\% de la fruta y el 69\% de los vegetales) (Benfield, Raimi \& Chen, 1999, pp. 29-89).

$\mathrm{El}$ agua es otro de los principales costes de la factura medioambiental. La proliferación de piscinas y zonas verdes hace que los suburbios consuman de cuatro a cinco veces más agua que los centros urbanos. Los habitantes de Tucson gastan 800 litros por persona y día, los de Albuquerque más de 1.000 y los de Phoenix más de 1.100. Todas estas ciudades están enclavadas en climas desérticos y su abastecimiento depende de corrientes subterráneas. Si tenemos en cuenta que la media pluviométrica de Las Vegas es de cuatro pulgadas anuales, podremos hacernos una idea aproximada de la dimensión del problema. El suministro de estas ciudades depende de macroproyectos infraestructurales. En los años 1930 se construyó la faraónica presa Hoover, que taponó el río Colorado con un dado de hormigón de 221 metros de altura por 200 de ancho. Esta descomunal obra de ingeniería consiguió que el desierto que comparten Arizona, Nevada y el sur de California dejara de ser un territorio inhóspito. También indicó la dirección de la única manera de sostener el 
modelo suburbano en el Sunbelt: la construcción de gigantescas y costosas obras de infraestructura. Gracias al Central Arizona Project, ejecutado en la década de 1980, Phoenix y Tucson aliviaron su necesidad de agua con un trasvase desde el río Colorado. Se cumplía así una de las reivindicaciones históricas del estado de Arizona que, en 1934, había llegado a declarar la guerra a California por este asunto.

Pero otras ciudades no fueron tan afortunadas. El Paso (Texas) no consiguió que Nuevo México le permitiese acogerse a una solución similar. Tras dar por perdida una batalla legal que se prolongó durante once años, acabó implementando medidas pioneras en el Sunbelt en cuanto al uso racional del agua. En 1991 aprobó una resolución transaccional que confiaba conseguir un suplemento de más de cien millones de $\mathrm{m}^{3}$ mediante el control de la evaporación de las reservas, la reducción del consumo per cápita a 600 litros/día, el aumento del flujo procedente de depuradoras, la compra y alquiler de parcelas de agua comunales y, principalmente, la reducción de las cuantiosas pérdidas que se producían en el transporte (Earl \& Czerniak, 1996, pp. 359-379). Otras ciudades optaron por ajustar el crecimiento urbano a la disponibilidad de agua (para Phoenix, por ejemplo, ese límite estaría en los siete millones de habitantes). Se producía así una paradoja: no era la tierra, sino el agua lo que ponía coto al crecimiento de las ciudades del Sunbelt (Golany, 1978).

Las facturas económica y medioambiental ponen de manifiesto la gran contradicción sobre la que se sostiene la riqueza de las ciudades del Sunbelt: el desarrollo suburbano es su razón de ser económica e ideológica, pero también una amenaza, especialmente en la economía globalizada, donde la calidad de vida se ha convertido en uno de los principales argumentos en la competencia entre ciudades. Hoy en día cuestiones como la degradación medioambiental, la escasez de agua, la obsolescencia de las infraestructuras o la contaminación pueden traducirse en desaceleración del crecimiento o, incluso, en decrecimiento.

La primera ciudad del Sunbelt donde esto se evidenció fue Los Ángeles. Durante ańos, estuvo transgrediendo la naturaleza que la rodeaba. Tras devorar los terrenos agrícolas del valle de San Fernando, el crecimiento suburbano se cebó con el condado de Orange, un vergel de naranjales. Le siguieron San Bernardino, los bosques de las estribaciones montañosas y, más recientemente, el desierto del Alto Mojave, cuya antesala (el valle del Antílope) fue engullida por un manto de piscinas y jardines. Es lo que Mike Davis (1998) denominó "la ecología del demonio". Este crecimiento provocó gravísimos problemas en las infraestructuras de la ciudad, incapaces de acompasar ese ritmo. En 1987, millones de litros de aguas residuales fueron vertidos a la bahía de Santa Mónica desde la planta depuradora de Hyperion, que no pudo absorber el espectacular desarrollo de las áreas urbanas a las que servía. A ello hubo que añadir la cuestión del abastecimiento de agua que, como hemos comentado, llevaba ańos enfrentando a California y Arizona. Por si fuera poco, Los Ángeles era una de las metrópolis más contaminadas del mundo desarrollado, debido a su altísima dependencia del automóvil. La ciudad no tenía capacidad financiera para hacer frente a este cúmulo de problemas. Cuando, en 1995, estuvo al borde de la bancarrota, la única salida que le quedó fue reducir drásticamente los servicios públicos que prestaba (clausurando treinta parques, quince bibliotecas y uno de los hospitales más importantes del país). El resultado fue que entre 1990 y 2000 
su crecimiento poblacional se redujo al $6 \%$. Y, lo que es peor, se generó una mala prensa que dio pie a que se acuñara un término que ha hecho fortuna como sinónimo de fracaso por congestión: "californication".

En el cambio de siglo, otras ciudades del Sunbelt parecían abocadas a seguir los pasos de Los Ángeles. A finales de la década de 1990, las infraestructuras de numerosas zonas de Atlanta mostraban síntomas de agotamiento. Como había ocurrido en el midtown de Houston, en varias de ellas el colapso del alcantarillado obligó a establecer moratorias de construcción. También era obvio que la red de suministro de agua no podría soportar los incrementos poblacionales previstos para los próximos ańos. La prensa hizo recuento: entre 1988 y 1998, habían sido taladas más de 75.000 hectáreas de bosques (la mayoría en la mitad norte del área metropolitana, donde se asentó el $70 \%$ de la población que Atlanta ganó en esa década). La dependencia del automóvil era absoluta, la ciudad ostentaba el récord nacional de kilómetros recorridos en los commutings: 56 en cada sentido. Ello se traducía en interminables atascos, un disparatado gasto de combustible y, lo más importante, una contaminación atmosférica que provocó problemas respiratorios a los ciudadanos. Al igual que ocurrió con Los Ángeles, estos datos erosionaron la reputación de Atlanta. En 2000, el Places Rated Almanac, una organización que clasifica las ciudades según la calidad de vida, hizo descender su posición desde el número uno, que ocupaba en 1981, al treinta y tres.

\section{Propuestas de corrección del modelo suburbano: "smart growth" y "edge cities"}

Los casos de Los Ángeles y Atlanta fueron catástrofes anunciadas. En los años 1970, las amenazas del crecimiento suburbano eran ya evidentes. De hecho, fue entonces cuando el gobierno norteamericano implementó una serie de leyes que, de una u otra manera, lo coartaban. En 1970 se aprobó la Clean Air Act (que establecía límites de polución ambiental); en 1972, la Clean Water Act (que obligaba a descontaminar ríos, arroyos y frentes marítimos); y en 1973, la Endangered Species Act (que paralizó la construcción de autopistas que atravesaban espacios naturales). La reacción gubernamental despertó la conciencia medioambiental de los norteamericanos. En 1998 se sometieron a referéndum 240 iniciativas antisuburbanización, que fueron aprobadas en un $72 \%$, fruto de lo cual resultaron más de mil enmiendas a las legislaciones estatales. A la cabeza de este movimiento estaban algunos estados del norte, como Maryland, Oregon y Nueva Jersey (Gillham, 2002, p. xiv).

En el Sunbelt el despertar fue más lento, pero logró cuestionar el consenso en torno a la ideología del crecimiento. La opinión pública se dividió en tres frentes: los que pensaban que todo debía seguir igual, defensores de la ideología del crecimiento; los que se oponían a cualquier tipo de crecimiento, grupos ecologistas radicales; y los que buscaban una solución intermedia. Estos últimos conformaron un movimiento que consiguió aglutinar a organizaciones de sesgo progresista, grupos empresariales conservadores y agencias públicas. Se trataba del "smart growth" ("crecimiento inteligente"), cuya máxima era: responder a la necesidad de crecer, pero limitando el impacto negativo. Lo que pretendían, por tanto, no era tanto negar la ideología del crecimiento, como reconducirla hacia parámetros sostenibles. 
Las cuestiones eran: cuánto, dónde y cómo crecer (Gillham, 2002; Lucy \& Philips, 2006; Williams, 2000).

La primera era difícil de refutar en términos genéricos: dependía de cada localidad. El smart growth recomendaba establecer cuotas máximas anuales, un tema delicado, ya que la Constitución estadounidense impide limitar el aumento demográfico de un territorio. Cualquier propuesta en ese sentido tan solo puede establecer ritmos y pautas justificadas por la incapacidad del estado de dotar el territorio de los correspondientes servicios e infraestructuras. Con respecto a la segunda, dónde crecer, aconsejaba prohibir urbanizar las zonas naturales de valor ecológico. Muchas de ellas estaban legalmente protegidas porque pertenecían a las administraciones federal y estatal, ${ }^{6}$ pero las que seguían en manos privadas habrían de ser compradas. Por último, al cómo crecer el smart growth respondía con un catálogo de propuestas claramente inspirado por la ciudad tradicional: mezcla de usos, alta densidad, transporte público, peatonalización, rehabilitación urbana y arquitectónica, viviendas sociales, etcétera. Las tres primeras constituían el núcleo duro del programa. La mezcla de residencias y oficinas, por ejemplo, podía reducir los commutings. Para ello reclamaba el establecimiento del patrón del "cuarto de milla": un radio de 400 metros alrededor de las viviendas (la distancia máxima que una persona admite hacer a pie en sus desplazamientos rutinarios), donde debían concentrarse los equipamientos. Por lo que respecta a la alta densidad, exigía parcelas más pequeñas y edificios más altos (en los entornos compactos disminuye el uso del automóvil y aumenta el del transporte público). En cuanto a este último, el smart growth proponía la creación de corporaciones de transporte de escala metropolitana o regional, primer paso para garantizar su eficiencia en un entorno suburbano. Contaba para ello con el apoyo de la Intermodal Surface Transportation Efficiency Act (ISTEA), una ley federal aprobada en 1991 que apuntaba hacia un cambio de paradigma en el transporte en Estados Unidos. Experiencias piloto fueron las del Hop Shuttle Service de Boulder (Colorado) y el Shuttle Bug Reverse Commute Project de Deerfield (Illinois), redes de minibuses de escala territorial. Pero la modalidad preferida por el smart growth para contrarrestar el uso del automóvil era el transporte ferroviario ligero. Las referencias, en este caso, provenían de San Francisco y su sistema BART (Bay Area Rapid Transit), y Boston y su мBTA (Massachusetts Bay Transportation Authority). Peter Calthorpe (1993) trasladó estas ideas a un modelo de suburbio que denominó TOD (Transit Oriented Development). San Diego (California) fue una de las primeras ciudades en estudiar su implementación.

Este último caso demuestra cómo el mensaje del smart growth también pareció calar en las ciudades del Sunbelt. A finales de 1990, el gobierno federal decidió suspender las ayudas a la construcción de autopistas en el área metropolitana de Atlanta. Esta moratoria no se levantaría hasta que no se diseñara un plan regional de transportes que redujera la contaminación a los niveles permitidos por la Clean Air Act. Así nació, en 1999, la Georgia Regional Transportation Authority (GRTA),

El gobierno central posee el 21\% del territorio de Estados Unidos, el 90\% del cual está situado en su mitad oeste. También los estados cuentan con amplias propiedades que les fueron otorgadas por el Congreso en 1787. 
una especie de gobierno metropolitano encargado de supervisar cualquier proyecto de urbanización que afectara al tránsito. Por lo que respecta a Los Ángeles, el alcalde pactó con los movimientos smart growth la implantación de 120 medidas encaminadas a cambiar los hábitos de producción y consumo. Por ejemplo, frente a la domesticación de la naturaleza vía mastodónticas obras de infraestructuras, se planteó la creación de ecosistemas capaces de absorber las aguas torrenciales. Además, se propuso la renaturalización del río Los Ángeles (Keil, 1998).

\section{FIGURA 5 | Edge cities en Dallas y Houston}
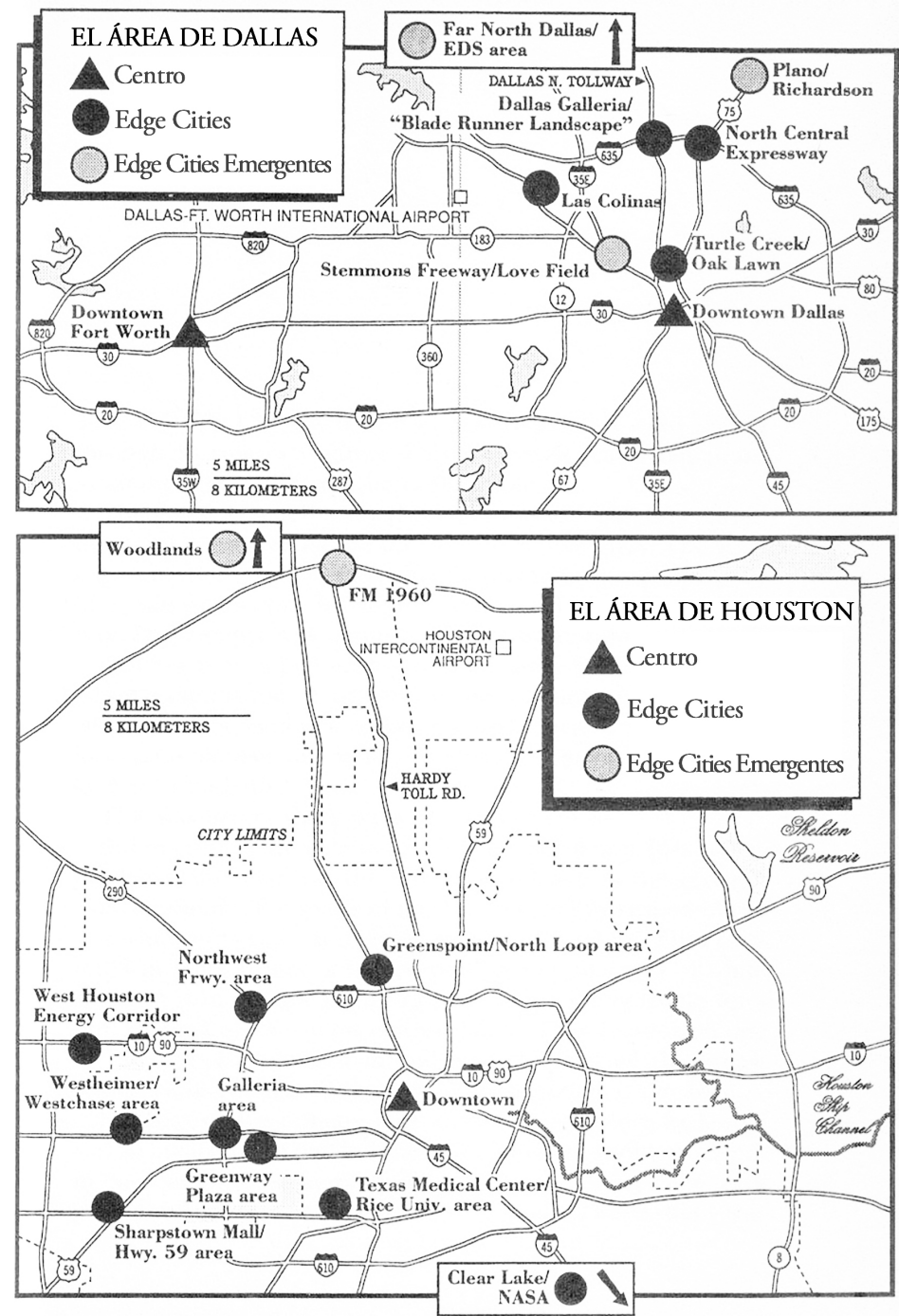

FUENTE J. GARREAU (I99I). EDGE CITY. LIFE ON THE NEW FRONTIER. NUEVA YORK: DOUBLEDAY, P. 2 I I (TRADUCCIÓN Y ADAPTACIÓN DEL AUTOR) 
Las "edge cities" fueron uno de los derivados del smart growth que más se propagó por Estados Unidos. Definidas por unos como "el mayor descubrimiento del urbanismo norteamericano desde 1980" (Sudjic, 1992, p. 116) y por otros como la principal transformación en la forma de hacer ciudades que se ha producido en Estados Unidos desde hacía siglos, las edge cities eran la penúltima generación de suburbios, si bien sus características formales y funcionales diferían tanto de las de los de posguerra que muchos las contemplaban como un fenómeno diferente.

Así opinaba Joel Garreau, autor del libro Edge City. Life on the new frontier e inventor de este término traducible como "ciudad de borde" (Garreau, 1991). Se trataba de suburbios de gran tamaño: de 4.000 a 10.000 hectáreas y 30.000 a 40.000 habitantes. Por un lado, eran encuadrables dentro del fenómeno de postsuburbia, ya que se situaban muy lejos de los centros urbanos, en áreas fronterizas entre la ciudad y el campo. Por otro, trasladaban a estos remotos territorios preceptos del smart growth. A diferencia del suburbio tradicional, en ellas se producía una rica mezcla de funciones que las convertía en ciudades autónomas. Joel Garreau afirmaba que en una edge city siempre había más puestos de trabajo que dormitorios. De hecho, no consideraba como tal a ninguna que no contuviera, al menos, $460.000 \mathrm{~m}^{2}$ de oficinas y $55.000 \mathrm{~m}^{2}$ de zonas comerciales. El segundo criterio smart growth asumido por las edge cities era el de la densidad. Sus parcelas eran de menor dimensión que las de los suburbios tradicionales y las viviendas unifamiliares convivían con bloques de apartamentos. Además, el crecimiento se producía en continuidad con el tejido ya construido, sin dejar espacios vacantes entre una fase y la siguiente (fenómeno denominado "salto a pídola"). Por último, las zonas de aparcamiento se concentraban en edificios en altura o en plantas subterráneas, evitando las playas de asfalto superficiales.

Las edge cities triunfaron en el Sunbelt. De acuerdo con el libro de Garreau, a comienzos de los años 1990 Dallas-Fort Worth y Atlanta contaban con siete de ellas; Houston con once, Phoenix con doce y Los Ángeles con veinticuatro (Garreau, 1992, pp. 426-439). Según Robert E. Lang, siete de ellas se encontraban entre las diez más "glamurosas" de Estados Unidos: Costa Mesa en Los Ángeles (con 2.600.000 $\mathrm{m}^{2}$ de oficinas), Post Oaks en Houston (2.000.000 m²), Cumberland Galleria en Atlanta $\left(1.600 .000 \mathrm{~m}^{2}\right)$, Perimeter Center también en Atlanta $\left(1.500 .000 \mathrm{~m}^{2}\right)$, LBJ Freeway en Dallas $\left(1.200 .000 \mathrm{~m}^{2}\right)$, Far North también en Dallas $\left(1.150 .000 \mathrm{~m}^{2}\right)$ y South Bay/LAx en Los Ángeles $\left(1.100 .000 \mathrm{~m}^{2}\right)$. Casi todas superaban en dimensión y población a los centros urbanos de sus respectivas áreas metropolitanas. Un ejemplo era The Woodlands, la mayor edge city de Houston en población (150.000 habitantes) y superficie (10.000 hectáreas, que contrastan con las 800 del downtown). Otro caso, en esta misma ciudad, era Post Oaks, en cuyos edificios de oficinas, algunos de ellos rascacielos como la Transco Tower, trabajaban 600.000 personas. Post Oaks era un auténtico "uptown", es decir, un distrito financiero alternativo al downtown (de hecho, su cámara de comercio había emprendido gestiones legales para denominarse como tal). 
FIGURA 6 | Dallas Galleria, edge city de Dallas

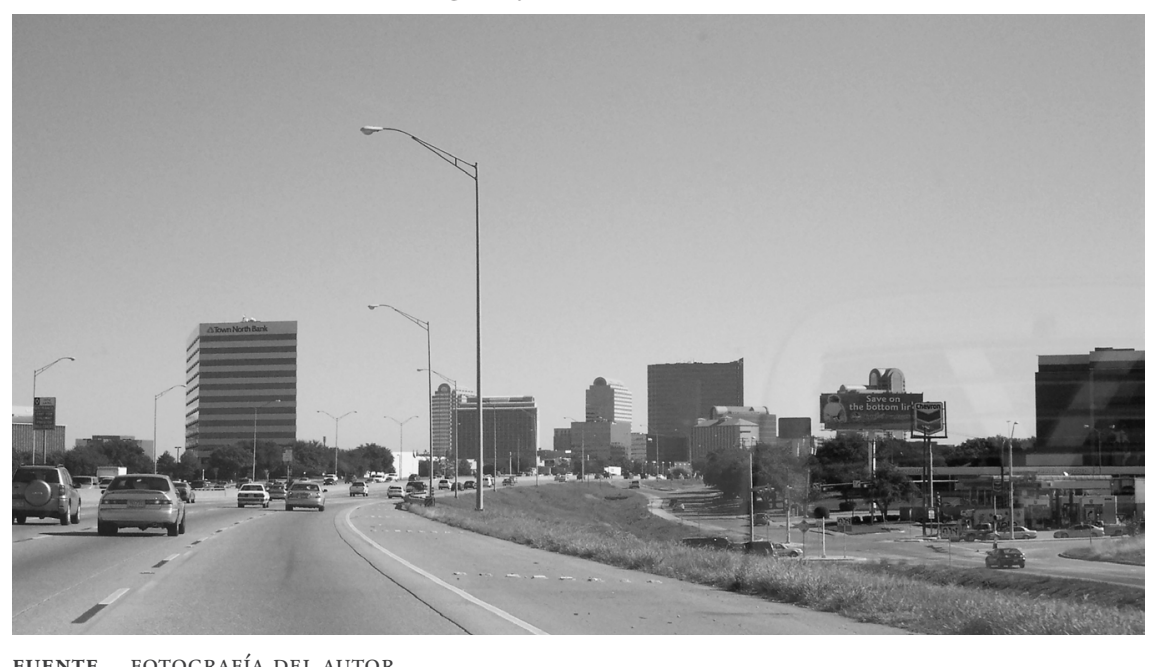

\section{La puesta en crisis del smart growth y la apuesta por seguir creciendo:} la "edgeless city"

A finales de la década de 1990 irrumpieron las críticas a las edge cities. Algunos académicos dudaban de que las esperanzas de conciliar crecimiento suburbano con sostenibilidad fueran realistas. Otros iban más allá y denunciaban que eran aliadas de las empresas automovilísticas y del petróleo. Para Richard Ingersoll (1994) no era casual que las cuatro principales de Houston estuvieran situadas a más de cuarenta kilómetros del downtown:

En Houston, conducir es un acto político; significa apoyar a la industria sobre la que se asienta la economía de la ciudad. Las urbanizaciones del extrarradio están tan íntimamente ligadas a la economía del petróleo que la relación no puede ser casual: Clear Lake y Kingwood fueron promovidas por la Friendswood Development Company, subsidiaria de Exxon; The Woodlands por Mitchell Energy; y el principal socio financiero de Gerald D. Hines en First Colony es Royal Dutch Shell. Por su parte, Mobil Oil está actualmente promoviendo once edge cities a lo largo y ancho del Sunbelt. Es obvio que es el interés de las industrias del automóvil y el petróleo el que induce estas promociones, que convierten la demanda de sus productos en un modo de vida (un viaje en torno a una de estas nuevas ciudades supone medio depósito de gasolina) (p. 11; traducción y adaptación del autor).

A comienzos del siglo xxi, Robert E. Lang (2003) se atrevía a certificar el declive de las edge cities. La razón no estaría tanto en sus desajustes con los postulados del smart growth, que, en todo caso, tan solo minaban su legitimidad ante la opinión pública, como en el agotamiento de los recursos que las hicieron competitivas en los años 1980. Ello empezó a ponerse de manifiesto cuando, al alcanzar la madurez, irrumpieron la 
congestión de tráfico, la polución ambiental y la especulación del suelo. Desaparecía así una de las principales bazas de las edge cities frente a los downtowns: los precios razonables. Según Lang, las empresas comenzaron a abandonarlas a finales de 1990.

El fracaso de uno de los estandartes del smart growth dio alas a los voceros de la ideología del crecimiento, articulados en organizaciones de todo tipo. La mayoría de ellas representaba a sectores económicos promotores de suburbios (como la National Association of Home Builders, la National Association of Realtors o la Heritage Foundation). En otros casos, ciertamente minoritarios, se trataba de grupos preocupados por la posibilidad de que las políticas smart growth provocaran un aumento del precio del suelo ${ }^{7}$ (como Habitat for Humanity). Unos y otros comenzaron a difundir datos y argumentos que pretendían contrarrestar el discurso del smart growth y su énfasis en la crisis medioambiental, a la que calificaban de exageración elucubrada por un exquisito círculo de intelectuales de izquierda. Para ello pusieron sobre la mesa una batería de contraargumentos.

El primero hacía referencia a la dilapidación del territorio. Las organizaciones pro suburbios matizaban: solo el 5\% de la superficie de Estados Unidos está urbanizada. Si continuara el actual ritmo de crecimiento, se necesitarían más de nueve mil ańos para agotar zonas como el desierto de Sonora. Tan solo los terrenos privados del condado de Maricopa (expoliados por las 0,4 hectáreas que le arrebata Phoenix cada hora) tardarían 336 años en urbanizarse. También en lo que respecta al territorio agrícola los defensores de la ideología del crecimiento insistían en desdramatizar. Según la National Association of Home Builders, su dimensión prácticamente no había variado en los últimos cincuenta ańos. En las zonas desérticas, además, esta actividad era perniciosa, ya que dilapidaba el líquido elemento (el 89\% del agua consumida en Arizona estaba destinada a usos agrícolas, lo que contrastaba con el $7 \%$ de las ciudades y el $4 \%$ de las industrias). Como hemos visto, en este tipo de entornos se daba una paradoja: una medida para ahorrar agua era recalificar las áreas rurales como suburbanas. Tucson ya la había puesto en práctica, consagrando a áreas suburbanas 4.500 hectáreas de cultivo. En lo referente a las especies animales, el contraargumento era que los suburbios eran más biodiversos que las tierras agrícolas. Según la Heritage Foundation, ciertas especies estaban aumentando de población gracias a ellos, como demostraba el hecho de que el crecimiento del número de ciervos de las áreas suburbanas de Nueva York casi triplicara la media del estado (610\% frente al 240\%). Entre los supuestos "beneficiarios" de la ideología del crecimiento también se encontraban los castores, los gansos, los zorros, los osos pardos, los pavos, los halcones y los coyotes. En cuanto a la contaminación, ponían sobre la mesa informes oficiales que afirmaban que la calidad del aire llevaba décadas mejorando en Estados Unidos. Entre 1982 y 1992, la polución ambiental bajó un $8 \%$ (a pesar del incremento de población y producción). Incluso en ciudades como Los Ángeles, denominada "la capital del smog", no había parado de decrecer desde 1950. Las organizaciones pro suburbios constataban, además, que la polución ambiental era mayor en las áreas urbanas densas, donde se concentran los atascos, el

7 En Phoenix el precio de la vivienda era un 35\% inferior al de Portland, la "capital del smart growth". 
gasto de gasolina y la emisión de residuos. Finalmente, cuestionaban el argumento del sobrecoste que suponía el modelo suburbano. Si bien era cierto que este exigía más kilómetros de redes, no lo era menos que era precisamente en los densos centros urbanos donde los precios se disparaban debido a los valores de suelo e instalación. Las calles, además, requerían un nivel de iluminación superior y mayor capacidad de recogida de aguas residuales, de abastecimiento de agua, etcétera.

Algunas de las organizaciones citadas constituyeron lobbies de presión para derogar las normativas antisuburbanización aprobadas desde $1970 .^{8} \mathrm{Su}$ trabajo, unido al apoyo político, económico y social con que contaba la ideología del crecimiento en el Sunbelt, explican que, a comienzos del siglo xxI, se expandiera por sus ciudades una nueva y extremadamente agresiva variante del modelo suburbano, a la que Robert E. Lang denominó "edgeless city" (en clara referencia a la superación de las edge cities). Ambas respondían al proceso de descentralización de las tareas de oficinas, pero desde el punto de vista morfológico eran opuestas (figura 7). Las edgeless cities eran extremadamente dispersas y amorfas. Se esparcían por enteras regiones metropolitanas ocupando los intersticios existentes entre suburbios y edge cities. Lo hacían, además, de manera casi imperceptible, con edificios modestos en apariencia y escala (de entre 5.000 y $6.000 \mathrm{~m}^{2}$ ), y separados entre sí por distancias enormes. Estas ciudades, por tanto, suponían un retorno a la baja densidad, a la dispersión, a la monofuncionalidad, a la dependencia del automóvil; en definitiva, a las esencias de la ideología del crecimiento.

\section{FIGURA 7 | Robert E. Lang: tipos de localización de edificios de oficinas}

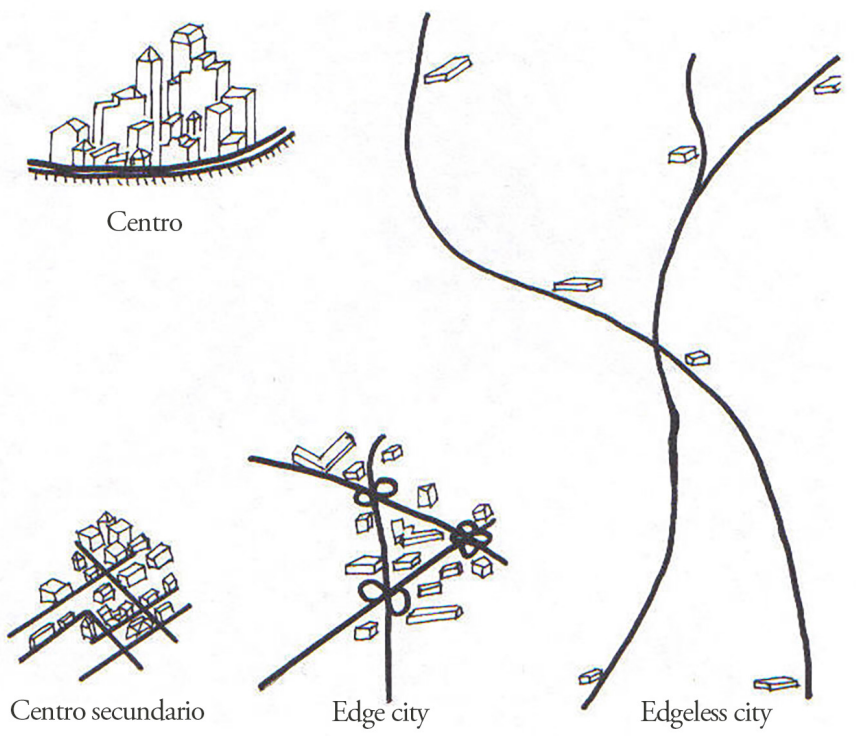

FUENTE R. E. LANG, (2003). EDGELESS CITIES. EXPLORING THE ELUSIVE METROPOLIS. WASHINGTON, DC: BROOKINGS INSTITUTION PRESS, P. 42 (TRADUCCIÓN Y ADAPTACIÓN DEL AUTOR) 
Lang defendía que, en 2003, la suplantación de las edge cities por edgeless cities ya se había producido. En ese año, las dos terceras partes del espacio de oficinas existente fuera de los downtowns de Estados Unidos estaban en edgeless cities y el tercio restante en edge cities. En términos generales, estas últimas tan solo albergaban el $25 \%$ de la superficie de oficinas del país: el otro $75 \%$ se lo repartían por igual downtowns y edgeless cities. En el Sunbelt las segundas sobrepasaban a los primeros: en Atlanta habían captado el 41,2\% de las oficinas y en Miami el 65,8\% (Lang, 2003, p. 55). Para Lang (2003), "el hecho de que las edgeless cities, que son tan desconcentradas, se hayan equiparado a los más importantes downtowns de los mayores mercados del país es asombroso. En las pasadas décadas ha ocurrido una revolución en la forma metropolitana. La jerarquía regional de las oficinas ha dado un vuelco" (p. 56; traducción y adaptación del autor).

Lang explicaba las razones de este vuelco apelando a las virtudes de las edgeless cities, entre las que destacaban la modestia y la flexibilidad. A sus promotores (normalmente empresas medianas y pequeñas dedicadas al back office) les era indiferente la localización urbana y la representatividad arquitectónica; se limitaban a construir edificios sencillos donde el suelo era barato. Este era su principal factor de competencia frente a downtowns y edge cities, pero no el único. Su carácter difuso no generaba congestión de tráfico y su práctica invisibilidad no molestaba a los NIMBY (“Not In My Back Yard"), personas acomodadas que vivían en suburbios y luchaban judicialmente por que nadie se asentase en el entorno de sus paraísos particulares (Logan, 1995). ${ }^{9}$

\section{Discusión}

Desde el punto de vista territorial, las edgeless cities indujeron un salto más allá de los siempre difusos límites de las ciudades del Sunbelt. Su bajísima densidad y extrema dispersión dieron lugar a la colonización de enclaves aún más alejados que los de las ya de por sí remotas edge cities, enclaves que, como ocurría en el caso de Atlanta, estaban a más de cien kilómetros del downtown. Las vistas satelitales lo demostraban. Las áreas metropolitanas del Sunbelt estaban licuándose unas con otras, traspasando fronteras estatales e internacionales. Las ciudades de la costa sur de California se habían fundido en un continuum urbano que enlazaba Santa Bárbara con Riverside, Los Ángeles, el condado de Orange, San Diego y Tijuana, ya en México. Algo similar ocurría en el área de Dallas-Fort Worth, de Houston (que avanzaba hacia Galveston), o de Atlanta (se calcula que, de seguir creciendo al ritmo actual, en 2050 la mitad de Georgia estará urbanizada).

Como comenta Edward W. Soja (2000), términos tan expansivos como "megalópolis" o "megaciudad" no bastan para definir esta realidad, donde se ha perdido todo foco y todo límite (p. 218). Algunos van más allá, y piensan que ni siquiera se les puede denominar "ciudad". Para Richard Skeates,

9 Algunos de los principales referentes (autores y trabajos académicos) de la lucha contra la ideología del crecimiento en las décadas de 1980 y 1990 fueron: Barnett (1995); Benfield, Raimi y Chen (1999); Calthorpe (1993); DeGrove (1992); Duany, Plater-Zyberk y Speck (2000); Ewing (2000); Katz y Scully (1993); Moe y Wilkie (1997); Orfield (1997). 
no podemos seguir usando el término ciudad en el sentido que lo hemos hecho hasta ahora, para describir una entidad que, aunque grande e inflada, es aún reconocible como una estructura limitada y circunscrita que ocupa un enclave específico. En su lugar hemos de lidiar con lo urbano: ni ciudad en el sentido clásico de la palabra, ni campo, sino un monstruo que lo devora todo y que se está tragando tanto a la ciudad como al campo y, al hacerlo, acaba con la vieja distinción entre ambos (en Graham \& Marvin, 2001, pp. 116, 121; traducción y adaptación del autor).

Quizás esta sea la gran “aportación” del Sunbelt. Algunos teóricos están convencidos de que el futuro de la ciudad americana se está forjando allí. Las razones que aducen apuntan a la sintonía existente entre la ideología del crecimiento y la lógica tardocapitalista: conservadurismo político, ultraliberalismo económico, imperio del sector privado, industrias tecnológicas, alta movilidad, etcétera. Más discutible es pensar que se trate de un modelo exportable a escala global o, incluso, nacional. La resistencia demostrada por la ideología del crecimiento tiene mucho que ver con las especificidades del Sunbelt: su corta historia, la inestabilidad de su tejido urbano y arquitectónico, el imperio de la cultura del automóvil y una desregulación urbanística tradicionalmente puesta al servicio de las elites económicas.

\section{Referencias bibliográficas}

Abbott, C. (1981). The new urban America. Growth and politics in Sunbelt cities. Chapel Hill, NC: The University of North Carolina Press.

Abbott, C. (1993). The metropolitan frontier. Cities in the modern American West. Tucson, Az: The University of Arizona Press.

Banham, R. (1971). Los Angeles. The architecture of four ecologies. Londres: Allen Lane.

Barnett, J. (1995). The fractured metropolis: Improving the new city, restoring the old city, reshaping the region. Nueva York: Harper Collins.

Benfield, F. K., Raimi, M. D. \& Chen, D. D. T. (1999). Once there were greenfields. How urban sprawl is undermining America's environment, economy and social fabric. Nueva York: Natural Resources Defense Council.

Bernard, R. M. \& Rice, B. R. (1983). Sunbelt cities. Politics and growth since World War II. Austin, Tx: University of Texas Press.

Calthorpe, P. (1993). The next American metropolis: ecology, community, and the American dream. Princeton, NJ: Princeton Architectural Press.

Castells, M. (1995 [1989]). La ciudad informacional. Tecnologias de la información, reestructuración económica y el proceso urbano-regional. Madrid: Alianza Editorial.

Cuff, D. (2000). The provisional city. Los Angeles stories of architecture and urbanism. Cambridge, MA / Londres: The miт Press.

Davis, M. (1998). Ecology of fear. Los Angeles and the imagination of disaster. Nueva York: Metropolitan Books Henry Holt and Cia.

DeGrove, J. M. (1992). The new frontier for land policy: planning and growth management in the States. Cambridge, MA: Lincoln Institute of Land Policy. 
Diamond H. \& Noonan P. (1996). Land use in America. Washington DC: Island Press.

Duany, A., Plater-Zyberk, E. \& Speck, J. (2000). Suburban Nation. The rise of sprawl and the decline of the American dream. Nueva York: North Point Press.

Earl, R. A. \& Czerniak R. J. (1996). Sunbelt water war: The El Paso-New Mexico water conflict. The Social Science Journal, 33(4), 359-379. https://doi.org/10.1016/S03623319(96)90012-9

Ewing, R. (2000). Pedestrian- and transportation-friendly design: a primer for smart growth. Estados Unidos: Smart Growth Network. https://www.epa.gov/sites/production/files/ documents/ptfd_primer.pdf

Fairbanks, R. B. \& Underwood, K. (eds.) (1990). Essays on Sunbelt cities and recent urban America. Arlington, Tx: Texas A\&M University Press.

Fishman, R. (1987). Bourgeois utopias. The rise and fall of suburbia. Nueva York: Basic Books.

Gammage, G. Jr. (1999). Phoenix in perspective. Reflections on developing the desert. Tempe, AZ: Arizona State University.

García Vázquez, C. (2011). Antípolis. El desvanecimiento de lo urbano en el Cinturón del Sol. Barcelona: Gustavo Gili.

Garreau, J. (1991). Edge City. Life on the new frontier. Nueva York: Doubleday.

Gillham, O. (2002). The limitless city. A primer on the urban sprawl debate. Washington, DC: Island Press.

Golany, G. (ed.) (1978). Urban planning for arid zones. American experiences and directions. Nueva York (NY) / Chichester (RU) / Brisbane (Australia) / Toronto (Canadá): John Wiley \& Sons.

Graham, S. \& Marvin, S. (2001). Splintering urbanism. Networked infrastructures, technological mobilities and the urban condition. Londres / Nueva York: Routledge.

Hayden, D. (2003). Building suburbia. Green fields and urban growth, 1820-2000. Nueva York: Pantheon Books.

Huxtable, A. L. (1978). Kicked a building lately? Nueva York: Quadrangle Books.

Ingersoll, R. (1994). Utopia limited: Houston's ring around the beltway. Cite. The architecture and design review of Houston, 31, 10-17. http://offcite.org/wp-content/uploads/ sites/3/2010/03/UtopiaLimited_Ingersoll_Cite31.pdf

Katz, B. \& Lang, R. E. (eds.) (2003). Redefining urban \& suburban America. Evidence from Census 2000. Washington, DC: Bookings Institution Press.

Katz, P. \& Scully, V. (1993). The New Urbanism. Toward an architecture of community. Nueva York: McGraw-Hill.

Kay, J. H. (1997). Asphalt nation. How the automobile took over America and how we can take it back. Berkeley, CA / Londres: University of California Press.

Keating, L. (2001). Atlanta. Race, class and urban expansion. Filadelfia, PA: Temple University Press.

Keil, R. (1998). Los Angeles. Globalization, urbanization and social struggles. Chichester, RU: John Wiley \& Sons.

Kling, R., Olin, S. \& Oster, M. (1995). Postsuburban California: The transformation of Orange County since World War II. Berkeley, Ca / Londres: University of California Press.

Kunstler, J. H. (1993). The geography of nowhere. The rise and decline of America's man-made landscape. Nueva York: Simon \& Schuster. 
Kunstler, J. H. (1996). Home from nowhere. Remaking our everyday world for the twenty-first century. Nueva York: Simon \& Schuster.

Lacayo, R. (1999). The brawl over sprawl. Times Magazine, 153(11), 44-48. http://content. time.com/time/magazine/article/0,9171,21490,00.html

Lang, R. E. (2003). Edgeless cities. Exploring the elusive metropolis. Washington, DC: Brookings Institution Press.

Logan, M. F. (1995). Fighting sprawl and city hall. Resistance to urban growth in the Southwest. Tucson, Estados Unidos: The University of Arizona Press.

Luckingham, B. (1989). Phoenix. The history of a Southwestern metropolis. Tucson, Az: The University of Arizona Press.

Lucy, W. H. \& Philips, D. L. (2006). Tomorrow's cities, tomorrow's suburbs. Chicago, IL / Washington, DC: American Planning Association.

Marshall, A. (2000). How cities work. Suburbs, sprawl, and the roads not taken. Austin, TX: University of Texas Press.

Moe, R. \& Wilkie, C. (1997). Changing places: rebuilding community in an age of sprawl. Nueva York: Henry Holt.

Orfield, M. (1997). Metropolitics: a regional agenda for community and stability. Washington DC: Brookings Institution and The Lincoln Institute of Land Policy.

Perry, D. C. \& Watkins, A. J. (eds.) (1977). The rise of the Sunbelt cities. Beverly Hills, CA / Londres: Sage Publications.

Scardino, B., Stern, W. F. \& Webb, B. C. (2003). Ephemeral city. Cite looks at Houston. Austin, TX: University of Texas Press.

Soja, E. W. (2000). Postmetropolis. Critical studies of cities and regions. Oxford, RU: Blackwell Publishers.

Squires, G. D. (2002). Urban sprawl. Causes, consequences \& policy responses. Washington, DC: The Urban Institute Press.

Sudjic, D. (1992). The 100 miles city. Orlando, fL: Harcourt Brace \& Company.

Swaback, V. D. (1997). Designing the future. Tempe, AZ: Arizona State University.

Williams, D. C. (2000). Urban sprawl. A reference handbook. Santa Bárbara, CA: ABC-CLIO. 